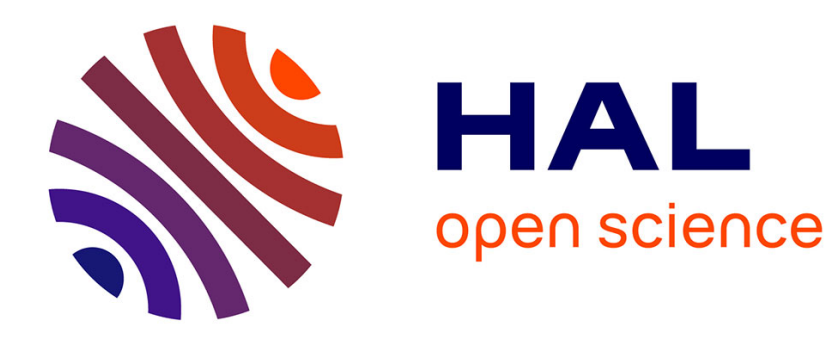

\title{
L'énigme des relations sino-américaines
}

Jean-Luc Domenach

\section{To cite this version:}

Jean-Luc Domenach. L'énigme des relations sino-américaines. Quaderni, 2003, 50-51, pp.211-229. 10.3406/quad.2003.1229 . hal-01018050

\section{HAL Id: hal-01018050 \\ https://hal-sciencespo.archives-ouvertes.fr/hal-01018050}

Submitted on 3 Jul 2014

HAL is a multi-disciplinary open access archive for the deposit and dissemination of scientific research documents, whether they are published or not. The documents may come from teaching and research institutions in France or abroad, or from public or private research centers.
L'archive ouverte pluridisciplinaire HAL, est destinée au dépôt et à la diffusion de documents scientifiques de niveau recherche, publiés ou non, émanant des établissements d'enseignement et de recherche français ou étrangers, des laboratoires publics ou privés. 


\section{L'énigme des relations sino-américaines}

In: Quaderni. N. 50-51, Printemps 2003. Images de l'Amérique du Nord vues par elle-même ou vues par les autres pp. 211-229.

Citer ce document / Cite this document :

Domenach Jean-Luc. L'énigme des relations sino-américaines. In: Quaderni. N. 50-51, Printemps 2003. Images de l'Amérique du Nord vues par elle-même ou vues par les autres pp. 211-229.

doi : $10.3406 /$ quad.2003.1229

http://www.persee.fr/web/revues/home/prescript/article/quad_0987-1381_2003_num_50_1_1229 


\section{Dossier}

\section{l'énigme \\ des relations}

sino-américaines

\author{
Jean-Luc \\ Domenach
}

Directeur de recherche

à la Fondation Nationale

des Sciences Politiques

Responsable de la recherche

expérimentale en sciences

humaines et sociales à Pékin

Après avoir été clairement définie durant les années de guerre froide (d'abord par l'hostilité puis par la coopération stratégique), la relation entre la Chine et les États-Unis est devenue un facteur obscur des relations internationales contemporaines. Sans doute ces relations ont-elles acquis une substance économique, sociale et même politique : les deux pays se connaissent mieux. Pour autant, ils ne se comprennent guère et l'obsession chinoise des États-Unis n'est pas dépourvue de dangers.

Commençons tout de même par une précision. Entre les États-Unis et la Chine d'aujourd'hui, le terme de "jeu" paraît plus approprié que celui de "nouvelle guerre froide", qui a encombré les journaux occidentaux. Car les relations américanochinoises d'aujourd'hui sont bien moins importantes pour l'équilibre du monde que l'ont été autrefois les rapports américanosoviétiques : elles sont trop déséquilibrées pour constituer un clivage central. Les ÉtatsUnis sont, pour parler comme Hubert Védrine, "l'hyper-puissance" du monde d'après la guerre froide, alors que la Chine est un pays, immense certes, mais qui n'a pas achevé son développement. En outre, la Chine ne possède pas l'attractivité nécessaire à la formation d'une alternative globale et d'un bloc d'alliances. 
En second lieu, ces relations ne peuvent être seulement définies comme un conflit, même si elles en portent le risque. Par leur histoire et leur contenu présent, ce sont des relations où l'amour et la haine se mêlent à l'indifférence et à l'incompréhension, où les maladresses précèdent $d$ 'intelligentes négociations, où les compromis ont toujours succédé aux tensions, et qui sont gravement compliquées par le rôle des opinions intérieures.

\section{Incompréhensions}

L'histoire des relations sino-américaines n'est pas tout uniment une histoire de haine. Au temps du dépècement de la Chine, les Américains sont en général restés en seconde ligne, marquant même par moments des désaccords avec le système des concessions; durant la Deuxième Guerre mondiale, elles ont aidé les deux camps en lutte contre le Japon avant d'imposer la place permanente de la Chine au Conseil de sécurité de la nouvelle Organisation des Nations Unies en 1945. Les Américains d'aujourd'hui admettent donc mal d'être considérés par les Chinois comme les parangons d'un Occident prédateur.

Mais ceux-ci se souviennent du rôle que les États-Unis ont tenu en Extrême-Orient dans la première partie de la guerre froide, et en particulier du soutien qu'ils ont réservé au camp nationaliste au cours de l'ultime guerre civile (1946-1949), puis des terribles combats que leurs armées de "volontaires" ont conduits en Corée sous les bombardements de l'aviation américaine (1950-1953). L'opposition à l'impérialisme devient alors pour le PCC une légitimation politique centrale. Les Américains, eux, considèrent la Chine comme leur ennemi majeur en Asie : ils protègent le régime Guomindang à Taiwan, puis interviennent au Viêt-nam, où leur escalade menacera vers 1965-1968 de s'étendre à la Chine. De part et d'autre, c'est le temps de l'obsession hostile.

Cette obsession réciproque empêche les observateurs de discerner à temps les nuances qui annoncent un changement. Alors que certains Chinois déçus par le communisme commencent à idéaliser l'Amérique, Mao apprend l'anglais avec une méthode Assimil au bord de sa piscine de la Cité interdite. Imagine-t-il de renouer avec Washington quand il rompt avec Moscou en 1959 ? En tout cas, il sait déjà que la vraie grande puissance, ce sont les États-Unis. De leur côté, bien qu'ils n'aient jamais coupé les communications avec Pékin, les dirigeants américains ont mis du temps à saisir l'opportunité ouverte par le conflit sino-soviétique, d'où des regrets qui expliquent en partie certaines patiences 
ultérieures. C'est seulement lorsqu'ils s'efforcent de limiter les effets de leur retrait du Viêt-nam que Nixon et Kissinger entendent les avances d'une direction chinoise qui s'inquiète de la menace militaire soviétique : et c'est leur fameuse mission en Chine de février 1972, conclue par le célèbre communiqué de Shanghai.

Ce renversement politique est l'acte fondateur des nouvelles relations sinoaméricaines jusqu'à nos jours. Les deux pays éprouvent alors leur aptitude à conduire des relations stratégiques qui dépassent les différences d'idéologie. Au reste, sur le moment, l'un et l'autre y trouvent des avantages concrets : du côté américain, une amitié nouvelle sur les arrières de l'URSS et du Viêt-nam, sans coût majeur pour la protection de Taiwan et pour les autres alliances asiatiques ; du côté chinois, une garantie de sécurité en pointillé face à l'URSS, la porte grande ouverte sur d'utiles échanges stratégiques et - Zhou Enlai l'espère - économiques avec le monde capitaliste.

Après l'arrivée au pouvoir de Deng Xiaoping, les relations sino-américaines sont développées par l'accord de normalisation des relations diplomatiques de 1979. Trois ans plus tard est signé un important accord sur les ventes d'armes américaines à Taiwan de 1982, par lequel les États-Unis s'engagent à plafonner puis à diminuer leurs livraisons militaires à condition que la Chine conduise une politique de réunification "pacifique". Les deux parties entretiennent des consultations militaires et combinent leurs efforts contre l'influence soviétique en Asie, notamment au Cambodge (où elles soutiennent longtemps les Khmers rouges contre l'occupant vietnamien). Les relations bilatérales, de plus en plus nourries, jouent un rôle essentiel pour la réussite de la politique de modernisation de Deng Xiaoping et pour le retour de la Chine dans le monde.

Ce bon départ cache cependant une dissymétrie et des malentendus qui préparent un avenir plus difficile. La dissymétrie est évidente : les relations entre les deux pays sont beaucoup moins importantes pour la super-puissance américaine que pour une Chine qui a grand besoin du commerce et de la technologie américaines; en outre, après 1985 quand l'URSS de Gorbatchev baisse le ton, l'amitié chinoise devient moins nécessaire pour les États-Unis alors que de son côté la Chine renoue avec le camp soviétique déclinant - cette double évolution affaiblit inévitablement la relation stratégique fondée en 1972. 
De cette dissymétrie découlent maladresses et déceptions. Nombre de responsables américains montrent au vieil Empire chinois tout juste renaissant une sympathie fort paternaliste. Ils y sont encouragés par la passion philo-américaine qui saisit alors la population citadine chinoise. Alors, en effet, se fait jour ce que l'on pourrait appeler le rêve américain de la Chine. Mais la réalité résiste : les Américains sont durs en affaires, et le massacre de Tiananmen rappelle que l'Amérique est bien lointaine.

Chez les responsables, la déception est surtout du côté de Washington - les dirigeants chinois, eux, ne se faisaient pas tant d'illusions. Surtout occupés par les événements d'Europe centrale et orientale, les diplomates américains avaient longtemps cru que la transition économique chinoise engendrerait inévitablement la libéralisation du régime de Pékin. Le massacre de juin 1989 sur la place Tiananmen est donc pour eux une brutale révélation : un régime qui foule aux pieds les droits de l'homme ne peut être un ami des États-Unis.

Au demeurant, du fait de l'effondrement de l'Union soviétique et de leur nouveau statut de seule super-puissance, les États-Unis se sentent bientôt en situation de mettre à la raison des "États brigands", comme l'Irak et pourquoi pas demain la Chine.
Les années 1989-1991 ont donc marqué un tournant dans les relations sino-américaines. Du point de vue américain, comme l'a montré le sinologue Michael Oksenberg, elles sont passées d'un statut par principe spécial à un autre, sujet à débat ; d'un alignement stratégique recherché à un rapport caractérisé à la fois par la coopération et les querelles; d'une définition admise par la majorité des Américains à une définition contestée et à une pratique influencée par les factions politiques, les lobbys économiques et les mouvements d'opinion ; mais aussi d'une situation où les intérêts économiques comptaient peu à une autre où ils sont devenus puissants ${ }^{1}$. Côté chinois, le changement a été parallèle mais plus accentué : la méfiance est devenue plus vive chez certains dirigeants et une large partie de la population ; mais la nécessité de développer des relations économiques nourries est plus fortement ressentie chez d'autres responsables. Des deux côtés, en tout cas, les rapports communs suscitent discussions et divisions

\section{Oscillations}

Ainsi s'expliquent largement les oscillations qui ont marqué depuis plus d'une décennie ce que l'on est tenté d'appeler aujourd'hui "l'affaire sino-américaine", au sens où il y a des affaires d'amour et de haine entre les 
individus. Des deux côtés se sont manifestées des hésitations et des divergences qui en certaines occasions ont paru remettre en cause la continuité et la cohérence des politiques.

En voici un historique simplifié. Après juin 1989 , les sanctions politiques décidées contre la Chine en accord avec les autres responsables occidentaux sont incomplètement appliquées puis tournées par les principaux pays asiatiques avant d'être abandonnées à partir de 1991. Bill Clinton arrive à la présidence en 1993 avec un programme hostile à Pékin. Les disputes commerciales se multiplient - la Chine se voit tout de même attribuer le bénéfice de la "clause de la nation la plus favorisée", sous conditions dès juin 1993, puis définitivement l'année suivante' ${ }^{2}$. Un incident naval survient en octobre 1994³ . En 1995-1996, ce sont les relations stratégiques qui se tendent : les mises en garde américaines ne détournent pas Pékin de lancer une campagne d'exercices militaires face à Taiwan, si bien qu'au début 1996 Washington doit envoyer deux porte-avions dans le détroit - certains observateurs estiment que les deux pays auraient alors été près du conflit militaire.

En 1997-1998, la nécessité de vivre ensemble l'emporte. Jiang Zemin se rend aux États-
Unis en octobre 1997 et Bill Clinton en juin suivant à Pékin, où il prononce une conférence sur les droits de l'homme : des deux côtés, c'est l'euphorie. Les dossiers commerciaux progressent et, au cours d'une conversation publique à Shanghai, Bill Clinton affermit son adhésion au principe d'une seule Chine en souscrivant à "trois non". Mais les problèmes et les méfiances demeurent et les relations se tendent à l'occasion de deux incidents militaires successifs : le bombardement par l'aviation américaine de l'ambassade de Chine à Belgrade en mai 1999, puis en avril 2001 un incident aérien entre un avion-espion américain et une patrouille de chasse chinoise en mer de Chine du Sud - un espace dont la Chine cherche depuis des années à faire "une nouvelle zone de nonintrusion américaine" dans la région limitrophe4.

En janvier 2001, l'arrivée aux affaires du président George W.Bush paraît d'abord annoncer un véritable gel de la relation sinoaméricaine. Durant sa campagne électorale, ce conservateur a en effet critiqué les faiblesses et les hésitations de Clinton à l'égard de Pékin. "Il faut traiter la Chine comme elle le mérite", déclare le nouveau secrétaire d'État Colin Powell. Ce n'est pas un "partenaire stratégique", plutôt "un compétiteur et un rival politique régional 
potentiel, mais aussi un partenaire commercial désireux de coopérer avec nous dans certaines zones"s. C'est en partie contre la menace chinoise que les dirigeants américains pensent alors réaliser le projet de bouclier anti-missiles dont ils ont convaincu les Japonais et auquel ils imaginent d'associer les Coréens voire les Taiwanais. Et George Bush autorise en juin 2001 des ventes d'armes très modernes à Taiwan'.

À l'heure où la négociation de l'entrée de la Chine à l'OMC approche de sa conclusion, le discours beaucoup plus dur de la présidence américaine fait craindre un "recouplage" entre les dossiers des droits de l'homme et des relations politiques, et plus généralement entre les dossiers politiques et économiques. Les dirigeants chinois n'ont donc pas intérêt au prolongement de la crise. Leur propagande publie des phrases apaisantes de Jiang Zemin' . La diplomatie américaine rétablit le contact : Colin Powell, le secrétaire d'Etat, se rend à Pékin en juillet et la Chine redevient un "partenaire"s.

Et puis, Pékin profite avec une extrême rapidité de l'aubaine politique que représentent dans un premier temps les attentats du 11 septembre 2001. Jiang Zemin ne tient pas compte des prévisions hâtives de ceux - parfois proches de lui - qui voient dans l'événement le début de la fin de l'Empire. Il téléphone immédiatement au président américain pour lui dire sa "solidarité", et la Chine approuve la grande opération afghane contre Ben Laden en exprimant avec une discrétion variable les conditions politiques et juridiques qui accompagnent son soutien'. La rencontre entre George Bush et Jiang Zemin à l'occasion de la réunion de l'APEC à Shanghai en octobre rétablit une cordialité de façade ${ }^{10}$. Les deux pays ont alors intérêt à faire valoir le retour de l'amitié : les ÉtatsUnis, parce qu'ils subordonnent tout à la campagne contre le terrorisme, la Chine parce qu'elle entend tirer avantage en ExtrêmeOrient de l'inévitable distraction américaine $^{11}$. La rapide visite que George Bush accomplit à Pékin en février 2002 réchauffe encore le climat : les dirigeants chinois n'ont pas le choix après le virage pro-américain de la politique russe. En fait, Jiang Zemin prépare déjà ce qui dans son esprit sera pour lui le couronnement de ses treize ans de pouvoir : un voyage triomphal aux États-Unis en octobre, avec une réception dans le ranch privé du président américain, qui paraît aplanir les différends quelques semaines avant le $\mathrm{XVI}^{\mathrm{e}}$ Congrès du PCC où il remettra la plupart de ses fonctions. Cette fois, l'apparence des bonnes relations est rétablie, et la Chine peut 
passer pour un partenaire respecté des États-Unis. Alors que Jiang Zemin se targue d'un triomphe personnel, les Américains escomptent à bon droit que Pékin laissera faire l'opération qu'ils préparent contre l'Irak.

Pourtant, difficultés et divergences n'ont pas disparu. Des deux côtés, les opinions demeurent méfiantes. Pékin s'agace de 1" "unilatéralisme" américain et attend en fait que l'obsession anti-terroriste des Américains les entraîne à commettre un fauxpas. George Bush réitère la position classique de Washington en faveur d' "une seule Chine" mais n'abandonne rien de ses exigences en matière de non-prolifération et surtout de politique taiwanaise. Il n'hésite pas à exprimer sa profonde méfiance envers le régime chinois : "Dans une société libre, la diversité n'est pas le désordre. Le débat $n$ 'est pas une lutte. Et la dissension n'est pas la révolution"'?.

En fait, dans la nouvelle donne politique qui a suivi le 11 septembre, la relation sinoaméricaine est devenue un point d'interrogation. En partie seulement, car les deux partenaires sont d'accord pour maintenir leurs rapports à un haut niveau et pour les gérer avec prudence. Mais l'embellie de l'après-terrorisme est lourde d'arrière-pensées : la politique américaine est désormais très préoccupée d'assurer la défense de Taiwan et de rallier ses alliés traditionnels d'Asie à sa grande mobilisation anti-terroriste, au point que l'on ne voit plus très bien quel rôle elle réserve à la Chine ${ }^{13}$. Pékin, de son côté, est à l'affût de toute nouvelle possibilité du côté de Taiwan. Sa patience à l'égard de l'hégémonie américain est exclusivement dictée par l'intérêt, et la diplomatie chinoise attend son heure.

\section{Méfiances et compromis}

Le contraste est aujourd'hui impressionnant entre le déploiement méfiant des diplomaties et la profondeur des malentendus d'un côté, la richesse des relations et le sérieux des compromis de l'autre.

Le fait est que les relations bilatérales entre les deux pays se sont développées et se développent encore avec un dynamisme qui n'a guère d'équivalents dans le monde actuel. Ce développement n'aurait pas été possible s'il n'avait été pensé par des conseillers sensés, promu par des dirigeants dans l'ensemble bien intentionnés et appliqué par de bons diplomates. Côté américain, conseillers, commentateurs et chercheurs n'ont jamais été à l'unisson, mais ils ont rarement proposé des initiatives extrêmes, et marquent en général du respect pour Pékin. Le fait que les études chinoises 
aux États-Unis soient de très loin les meilleures au monde n'a certes pas garanti la justesse constante de l'analyse, mais les sinologues qui ont conseillé la politique américaine ont tout fait pour servir, et leur pays, et la cause des relations avec la Chine : on pense à des hommes comme Michael Oksenberg et, plus récemment, Kenneth Lieberthal. À Pékin, il s'est créé une tradition d'attention prioritaire à la politique américaine, qui fait que les meilleurs spécialistes des États-Unis sont de bonne qualité : par exemple Wang Jisi, le directeur de l'Institut des Etats-Unis à l'Académie des sciences sociales.

Après une époque où les présidents ne se rencontraient que de loin en loin, les contacts directs, voire même les conversations téléphoniques, sont devenus plus fréquents. George Bush Junior marque une prudence exceptionnelle en matière de relations avec la Chine : celles-ci constituent probablement la partie la plus "stratégique" de sa politique étrangère. De part et d'autre, les diplomates ont fait et font de leur mieux, avec d'inévitables variations dans l'habileté. Les nominations sont généralement bien intentionnées : ainsi, après l'accession au pouvoir du nouveau président américain, c'est un avocat d'affaires lié au commerce entre les deux pays qui a reçu l'ambassade de Pékin, alors que, du côté chinois, on nommait à Washington une vieille connaissance de George Bush Senior ${ }^{14}$. Ces facteurs aident à comprendre que les relations entre les deux pays aient été dans l'ensemble très maîtrisées politiquement. À Washington comme à Pékin, le président les considère comme de son ressort éminent et fait les efforts nécessaires pour en conserver le contrôle, ce à quoi il parvient en général mais il $\mathrm{y}$ a eu des accrocs à la règle à cause du rôle des lobbys et des factions politiques aux États-Unis, de l'armée en Chine. De part et d'autre, aussi, des ordres sont donnés pour que les désaccords soient exprimés dans des termes contrôlés, et pour que les crises fassent l'objet d'un traitement diplomatique, même et surtout lorsqu'elles impliquent l'armée : ce fut le cas en 1999 (malgré le recours réminiscent de l'époque maoïste à la "colère des masses" à Pékin) et en 2001. Cette dernière crise, née en avril d'un incident aérien entre la chasse chinoise et un navire-espion américain par la suite arraisonné, qu'elle ait été ou non le résultat d'une provocation volontairement organisée par le côté chinois, comme l'assurent certains observateurs, aura été un cas exemplaire de traitement diplomatique à chaud d'un incident militaire.

\section{Le rapprochement économique et social}

En outre, le dossier économique est 
désormais le secteur le plus solide des relations bilatérales. Les investissements américains en Chine ont considérablement augmenté depuis 1995 et atteignent au moins 30 milliards de dollars ${ }^{15}$. Ils n'émanent pas seulement de Chinois américains, ni même de multinationales à la recherche d'une main-d'œuvre bon marché : c'est l'Amérique des Burger King et des Kentucky Fried Chicken comme d'IBM ou Motorola qui progresse en Chine, ce sont les banques, les assurances qui se préparent à l'ouverture du marché chinois.

Le commerce a progressé rapidement : de 33 milliards de dollars en 1992 à plus de 115 milliards en 2000, ce qui fait de la Chine le quatrième partenaire commercial des ÉtatsUnis $^{16}$. Celle-ci est à tous égards gagnante : elle reçoit la haute technologie indispensable à son développement (la moitié des exportations américaines en 1996), consacre aux États-Unis $40 \%$ de ses exportations et retire de ce commerce un excédent déterminant : plus de 80 milliards de dollars ! Du côté américain, les échanges impliquent des lobbys exportateurs influents, dont celui des céréaliers du Middle West ${ }^{17}$. Surtout, il suscite d'immenses espoirs dans l'avenir d'une Chine plus ouverte, où l'économie américaine pourrait trouver des marchés et engager des coopérations novatrices. Aussi étrange que cela paraisse, malgré le déséquilibre des échanges, les milieux d'affaires américains sont dans l'ensemble favorables à une politique positive envers Pékin.

Le développement des relations économiques favorise les rapports entre les sociétés. La petite communauté d'expatriés américains en Chine ne cesse d'augmenter, et les événements chinois font parfois les gros titres des médias d'Outre-Atlantique. Cependant, il reste beaucoup à faire pour que la Chine entre vraiment dans l'horizon des Américains : la faiblesse du tourisme en est un signe concret. Le rapprochement des sociétés s'opère donc surtout de la Chine vers les États-Unis. Ce sont d'abord, on l'a dit, des contingents d'étudiants chinois croissants depuis vingt ans : officiellement 60000 , en fait bien plus de 100000 d'entre eux séjournent actuellement sur les campus américains ${ }^{18}$. Seule une minorité est rentrée en Chine, mais il y a parmi eux des intellectuels fameux comme le sociologue $\mathrm{Hu}$ Angang, ancien docteur de Yale, ou encore les fils de Jiang Zemin, Zhu Rongji et $\mathrm{Li}$ Peng lui-même ${ }^{19}$. La majorité reste aux États-Unis, où ils réussissent en général fort bien et finissent par acquérir la nationalité américaine.

Ancienne, diversifiée et puissante, régulièrement renforcée par les arrivages 
successifs de victimes du communisme, la communauté chinoise des États-Unis reçoit depuis 1979 un filet constant de nouveaux arrivants. Dans leur majorité ce sont des clandestins, dont certaines affaires ont illustré le destin tragique : par exemple celle du Golden Venture, un cargo dont les 286 passagers se jetèrent à l'eau dans la baie de l'Hudson, parmi lesquels 40 périrent ${ }^{20}$. Mais l'administration américaine s'est par moments montrée plus large, pour des raisons politiques qui ont suscité le mécontentement de Pékin : ainsi, à partir de 1990 , les victimes de la répression de juin 1989 ont fait l'objet de mesures particulières, et à un moindre titre celles de la politique de l'enfant unique. Ces mesures ont été partiellement rapportées en 1993, mais l'immigration chinoise continue sous toutes les formes imaginables : elle aurait représenté 400000 personnes de 1982 à 1997 , et tout indique qu'elle se poursuit aujourd'hui' ${ }^{21}$.

Les autorités chinoises s'émeuvent périodiquement de l'écho que reçoivent aux États-Unis les dissidents chinois qui s'y sont regroupés, et en particulier Wei Jingsheng. Le gouvernement américain, pour sa part, demande à la Chine de combattre les "têtes de tortue" qui organisent l'émigration clandestine. Mais la communauté chinoise des États-Unis, forte aujourd'hui d'un à deux millions de personnes, est surtout une extraordinaire réussite : la réussite académique de jeunes gens si nombreux que les universités sont souvent contraintes de leur opposer un numerus clausus; et aussi leur réussite professionnelle dans des métiers nouveaux comme ceux dérivés de l'informatique, en particulier en Californie : dans la fameuse Silicon Valley, $17 \%$ des firmes électroniques fondées depuis 1980 sont aujourd'hui détenues par des Chinois d'origine ${ }^{22}$. Cette communauté constitue un lien entre les parties les plus dynamiques des deux pays.

Au-delà des méfiances et des rumeurs, sa prospérité fait voir l'attraction intense et durable ressentie par de larges fractions de la société chinoise pour les États-Unis, et l'accueil distrait sans doute, mais au total libéral que la société américaine réserve aux émigrés chinois.

\section{Amour et haine}

Malgré ce substantiel rapprochement, les sentiments sont devenus des deux côtés de plus en plus ambivalents. Le fait est particulièrement spectaculaire en Chine, notamment dans la jeunesse. En 1996, un sondage révélait en effet que les États-Unis étaient le pays le plus apprécié des jeunes Chinois (après Singapour) mais aussi le 
moins aimée $e^{23}$

Cette ambivalence, en partie liée à l'évolution de la scène chinoise, met en évidence un mélange d'amour et de haine difficile à analyser. On ne peut éviter de penser que bien des jeunes Chinois avaient d'abord trop aimé l'Amérique, que, devenus adultes, une déception s'est développée chez eux dans les années quatre-vingt-dix. Peut-être certains ont-ils transféré sur l'Amérique une partie de la déception qu'ils ressentaient à l'égard de leur propre pays après Tiananmen. Au reste, l'Amérique ellemême avait bien vite abandonné le mouvement démocratique chinois, et elle avait cru s'exonérer par un blocus qui était perçu comme un deuxième abandon. Le rêve changeait désormais de nature. Il fallait s'approprier tout ce qui pouvait l'être des ressources de cette immense puissance. Mais inutile d'espérer qu'elle aime et comprenne la Chine.

Bien des ignorances, des distractions et des grossièretés qui passaient auparavant pour des bizarreries scandalisaient. Un exemple. Lors d'un banquet offert à Pékin en 1997 à une centaine de membres du Congrès américain, l'un d'entre eux demanda benoîtement au vice-ministre qui les recevait : "Je voudrais seulement savoir si vous admettez Jésus Christ comme votre propre sauveur?"'Interloqué, celui-ci ne put que répondre sèchement: "Non"24. Combien y eut-il d'épisodes de ce type?

Cette Amérique à la fois désirée et détestable ne cessait de gagner en puissance. Mais elle n'aimait pas la Chine et entendait garder pour elle ses propres merveilles. Comme seule la Chine menaçait sérieusement son monopole, c'est inévitablement contre Pékin que Washington rassemblait ses forces : comment éviter cette conclusion? Une bonne partie de ses admirateurs tournèrent casaque entre deux séances de films américains.

Ce changement d'atmosphère ne déplaisait pas aux autorités chinoises. Depuis la fin de la guerre froide, l'anti-américanisme n'avait cessé de monter dans l'appareil chinois, de documents internes en pétitions d'officiers supérieurs et en ouvrages de stratèges plus ou moins bien en cour. En 1993, un livre à succès avait carrément imaginé une guerre avec les États-Unis -la mode se développerait plus tard de l'autre côtées . Au début de 1994, lors d'une "conférence de travail du Comité central", le chef d'étatmajor de l'APL, Zhang Wannian, avait violemment critiqué "l'hégémonisme américain", et le prudent Hu Jintao lui-même avait déclaré tout haut ce que beaucoup pensaient désormais : "Suivant la stratégie 
hégémoniste globale des États-Unis, leur principal rival est à présent la République populaire de Chine"26. Bientôt, c'est la presse officielle qui incriminait l'“arrogance", la "xénophobie", voire l' "impérialisme" des États-Unis.

La scène américaine avait elle aussi changé. Non que la Chine y ait jamais constitué une préoccupation majeure. L'immense majorité de la population américaine ne se préoccupe du monde chinois que par éclipses, mais la nouveauté est que dans ces moments-là elle s'en inquiète. Dans l'automne 1997, deux sondages concomitants indiquaient que $\mathbf{6 0}$ $\%$ de la population considéraient la Chine comme "un adversaire" ou "un problème sérieux"27. La critique de la situation chinoise en matière de droits de l'homme est de plus en plus générale : les médias réservent à la dissidence chinoise une attention par moments précise, et le Tibet du Dalai Lama inspire une mode qui s'étend jusqu'à Hollywood. Les dénonciations de la répression en Chine s'ajoutent aux critiques des pratiques commerciales chinoises et aux inquiétudes concernant l'ascension de la puissance.

Les autorités chinoises ont mis longtemps pour comprendre le rôle des groupes de pression hostiles, au point de les confondre parfois avec les organes officiels. Mais il est vrai que la Chine est devenue un vrai sujet pour les membres du Congrès, qui ont à plusieurs reprises gêné la politique de Bill Clinton. Et un sujet qui compte en temps d'élection : pour cette raison, Bill Clinton puis George Bush sont arrivés à la présidence précédés de promesses d'une politique plus rigoureuse envers Pékin. Pour cette raison, également, les autorités américaines ont souvent donné un ton moraliste à leur politique chinoise, en mettant sur la place publique des sujets que d'autres gouvernements abordent avec plus de discrétion : les droits de l'homme, les exportations de matériels militaires sensibles par Pékin ou ses efforts pour capter la haute technologie. Pour cette raison, enfin, Washington a laissé se développer une atmosphère d'espionite : divulgation des manœuvres des services chinois pour $s$ 'infiltrer dans les réseaux de financement de la campagne électorale de Bill Clinton en 1996 ; informations sur le "retournement" de plusieurs responsables militaires chinois ; rumeurs, rapports et procédures contre des cas d'espionnage supposé (dont le plus célèbre, qui a fait l'actualité en 1999, semble controuvé) $)^{28}$. Et Washington ne se contente pas de contre-espionnage. Ses systèmes d'observation du déploiement militaire chinois sont en alerte, pas toujours à bon escient : au début 2000 , ils auraient informé les alliés asiatiques des États-Unis 
d'un blocus imminent du port taiwanais de Kaohsiung ! ${ }^{29}$ Ils exaspèrent les dirigeants chinois, maintiennent en alerte leur aviation et provoquent de fréquents incidents, dont le plus grave a déclenché la crise d'avril 2001. De leur côté, les dirigeants de Pékin conduisent sur leur territoire une politique très subtile $d$ 'arrestation et de relaxe de chercheurs ou d'hommes d'affaires américain, de préférence Chinois d'outre-mer, et ils intensifient systématiquement leur effort de pénétration des milieux de la recherche scientifique américaine. Par moments, le climat des relations sinoaméricaines est devenu franchement détestable.

\section{Le grand jeu sino-américain}

Ces bisbilles s'expliquent par un problème beaucoup plus général qui ne laisse pas d'inquiéter : les immenses progrès accomplis depuis vingt ans par les relations bilatérales ne compensent pas complètement des divergences qui en dernière analyse proviennent désormais de désaccords globaux. Bien qu'elle soit partiellement éclipsée par la grande campagne antiterroriste, l'affaire sino-américaine est en passe de devenir un grand jeu qui intéresse l'avenir de la planète.

La divergence de fond date pour l'essentiel de la fin de la guerre froide, qui a modifié fortement la situation des deux puissances. Alors qu'elle apportait à Washington un statut d'unique super-puissance, elle portait une menace de mort pour les dirigeants chinois. En effet, elle coïncidait avec un effondrement quasi-général des systèmes communistes dont eux-mêmes avaient manqué de peu d'être victimes au printemps 1989. Elle accélérait aussi une mondialisation économique dont le cour se trouvait aux États-Unis et dont les effets idéologiques paraissaient traverser les frontières des États.

Ces tendances furent d'emblée perçues par Pékin comme des menaces pour l'indépendance de la Chine et pour son régime politique lui-même : le danger n'était plus seulement extérieur, mais intérieur, il n'était plus seulement politique mais idéologique. Un fossé séparait désormais les intérêts stratégiques des deux pays : d'un côté, ceux d'une super-puissance qui se sentait moins contrainte d'admettre la différence politique ; et de l'autre, ceux d'un régime qui entendait conserver sa personnalité et qui affirmait sa candidature à plus de puissance.

Mais les dirigeants de Pékin avaient suffisamment de lucidité pour comprendre que, l'option "révolutionnaire" et celle de la 
"capitulation" étant également écartées, ils n'avaient d'autre solution que d'emprunter une voie moyenne : grâce à de bons rapports bilatéraux avec les États-Unis, entrer dans le marché mondial pour accélérer l'ascension économique du pays, mais en maintenant et si possible en accroissant son indépendance politique et stratégique.

À partir de 1992 (fin du blocus occidental et relance de l'ouverture chinoise) et surtout de 1994-1995 (arrivée au pouvoir effectif de Jiang Zemin), Pékin a donc entrepris une véritable campagne destinée à progresser sur deux bords à la fois : assurer des rapports suffisamment nourris avec les États-Unis pour protéger sa croissance et intensifier son ouverture au marché mondial, mais renforcer tout autant la souveraineté du pays et le niveau des interventions de sa diplomatie. La marche a été difficile et son trajet parfois hésitant, car il dépendait à la fois des circonstances intérieures et des relations avec Washington. Mais la stratégie s'est révélée dans l'ensemble victorieuse, en bonne partie car la diplomatie chinoise a très intelligemment mis en œuvre les cartes politiques, économiques et psychologiques dont elle disposait dans le reste du monde, tout particulièrement en Asie.

À partir de 1998, les bonnes nouvelles se sont accumulées sur le front extérieur. La première a été la victoire de l'économie chinoise sur la crise asiatique, qui signifiait que l'économie chinoise était de plus en plus une force et non une faiblesse, et que le statut régional de la Chine était en voie d'affirmation - un phénomène évident après la reprise de Hong Kong en 1997 puis de Macao en 1999, qui éclipsait quelque peu les échecs des pressions sur Taiwan.

La seconde bonne nouvelle a été la négociation victorieuse de l'entrée de la Chine à l'OMC (novembre 2001): victorieuse, parce qu'elle était conclue dans des termes favorables, mais aussi parce qu'elle s'accompagnait d'une hausse nette de la "posture" de la Chine face à la superpuissance américaine. Cette dernière évolution était confirmée par ce qui est apparu d'abord comme une troisième bonne nouvelle pour Pékin, le terrible coup reçu le 11 septembre 2001 par l'Amérique de Bush, qui paraissait ouvrir à la Chine des hypothèses intéressantes: soit un enlisement des États-Unis dans un combat difficile, soit leur négligence des problèmes de l'Extrême-Orient.

Dans une atmosphère intérieure marquée par un nationalisme croissant, les dirigeants chinois paraissent aujourd'hui déterminés à mettre à profit ces progrès pour aller de l'avant. Mais ils doivent tenir compte des 
rapports de force et continuent à considérer que la modernisation du pays et son engagement dans le marché mondial demeurent des priorités absolues.

Pour ces raisons, ils n'ont guère changé l'analyse qu'ils font de l'évolution du monde depuis une dizaine d'années. Celuici est toujours compris comme travaillé par un mouvement de mondialisation dominé par les États-Unis, qui entraîne deux tendances contradictoires : des tendances à la "multipolarisation", et d'autre part des efforts récurrents de la super-puissance américaine pour les combattre. Tout en maintenant des rapports bilatéraux pacifiques avec la même super-puissance, la Chine se range naturellement dans le camp "multipolaire". Il existe donc une contradiction entre la Chine et les ÉtatsUnis. Mais elle n'est pas définie comme “antagoniste", et Pékin n'appelle pas les forces favorables à la multipolarité à former un "front", et cela fait la grande limite de la politique chinoise.

L'épisode anti-terroriste qui s'est développé depuis le 11 septembre 2001 n'a pas encore conduit Pékin à modifier cette analyse. Mais, si la Chine a réagi avec modération à l'événement, elle a été par la suite marginalisée diplomatiquement, alors que les Américains se montraient de plus en plus actifs tant en Russie et en Asie centrale qu'en Extrême Orient ${ }^{30}$. On peut donc se demander si elle ne sera pas prochainement conduite à hausser son niveau d'ambition, c'est-à-dire d'abord à durcir son attitude à l'égard des États-Unis.

\section{L'obsession américaine de la Chine}

Curieusement, cette focalisation sur les États-Unis constitue une grande faiblesse de la politique étrangère chinoise. Elle répond en effet à une fixation passionnée qui la prive d'une large partie des moyens adéquats.

Le fond des choses est que les Chinois s'intéressent trop aux États-Unis mais qu'ils les connaissent trop mal. Dans la vie quotidienne, tout témoigne de cette fixation, qui va jusqu'au ridicule : les milliers de restaurants Mac Donald's, la mode vestimentaire, les villas sur le modèle américain, les publicités télévisées, le triomphe des films américains (jusqu'à $70 \%$ des spectateurs), la fascination pour les études dans les grandes universités américaines. Les États-Unis sont le but rêvé de tous les migrants. S'il est un rêve commun à tous les citadins chinois, c'est bien celui de vivre comme aux États-Unis.

Ce rêve coexiste avec des ignorances et des 
incompréhensions également immenses. Les Chinois se précipitent vers la langue anglaise mais ne sont guère capables de la parler. Ils n'écoutent pas les récits plus nuancés des retours d'Amérique - sauf pour changer leur admiration en détestation. La presse est sans nuances et les livres sur l'Amérique sont le plus souvent d'une incroyable faiblesse. La Chine est fascinée par les États-Unis, mais elle les méconnaît. Il n'y a pas eu un seul Chinois pour croire que le bombardement de l'ambassade de Chine à Belgrade en mai 1999 ait pu être une erreur - une hypothèse que nombre d'Européens considéraient alors comme parfaitement plausible.

Il y aurait beaucoup à dire sur cette fascination. On se contentera ici d'indiquer que deux réflexes psychologiques au moins semblent à l'œuvre. Le premier, issu d'habitudes plus ou moins anciennes, consiste à considérer que la puissance résulte d'une perfection sinon morale du moins sociale, et il est souligné par la leçon des maîtres du nationalisme chinois contemporain (relayée à partir de 1980 par Deng Xiaoping) qui donnaient pour objectif à la nation chinoise de s'inspirer du meilleur Occident. Le deuxième réflexe est issu du malheur et de la misère. Les Chinois ont immensément souffert dans leur existence avant et sous Mao, et ils commencent tout juste à sortir d'une longue nuit. Ils en idéalisent d'autant plus le pays vainqueur de la guerre froide qui est aussi le plus riche du monde : pays merveilleux s'il se livre à eux, détestable s'il se refuse.

Cet état d'esprit a largement investi la politique étrangère chinoise -en partie parce que ses responsables d'aujourd'hui sont les anciens étudiants que Mao Zedong avait désespérés et contraints à la misère de l'exil durant la Révolution culturelle, en partie aussi parce que la politique étrangère chinoise est devenue plus fragile devant l'opinion. C'en est fini des politesses soigneusement calibrées d'autrefois : seuls comptent vraiment, les États-Unis (et le Japon comme repoussoir...). Non seulement les petits pays mais les grands pays du Tiers Monde, et même les vieilles nations d'Europe ne reçoivent plus qu'une attention seulement entrecoupée de quelques remarques surprises - par exemple celles que suscitent les brillantes négociations conduites par deux commissaires européens, le britannique Christopher Patten ou le français Pascal Lamy.

Cette atmosphère conduit la Chine à exagérer en faveur de Washington son analyse du rapport des forces dans le monde d'après la guerre froide. Elle contribue à expliquer la quasi disparition de la politique chinoise en 
direction du sud de la planète, au delà de quelques discours rituels; son incapacité récurrente à appliquer ses promesses de développer un véritable dialogue politique avec l'Union européenne et ses principaux membres; et plus largement son absence d'intérêt à peu près totale pour une approche plus collective des relations internationales.

Pékin ne manque certes pas d'excuses : par exemple la faiblesse au moins aussi prononcée des diplomaties russe, française ou allemande face à Washington. Mais celles-ci ont une riche expérience des rapports avec les États-Unis, et elles peuvent attendre de leur collaboration des avantages plus ou moins substantiels. Pour Pékin, au contraire, le face à face avec Washington porte des avantages dangereux en même temps que des inconvénients très forts.

Les avantages, immédiats et immenses, sont évidemment de nature économique, technologique et culturelle. Mais ils sont politiquement dangereux en ce qu'ils tendent à focaliser vers les États-Unis une partie importante de l'économie, de la science, de l'intelligence et plus précisément des élites chinoises : en cela, ils sont porteurs de graves conflits internes à l'intérieur de la politique chinoise, car le pouvoir de Pékin devra inéluctablement mettre le holà à ce qui apparaît à tous comme une américanisation en profondeur des forces vives du pays.

Les inconvénients sont énormes. En effet, le choix de la bilatéralité place la Chine en situation de faiblesse face à un pays qui, de son côté, la comprend au moins aussi mal et l'ignore plus encore. Il lui laisse le choix entre deux politiques également dangereuses. La première consistait, avant le 11 septembre, à altermer les provocations et les compromis : elle fut habilement conduite jusqu'au début de la grande mobilisation anti-terroriste. Depuis cette époque, les provocations sont devenues trop risquées, car la diplomatie américaine a changé de nature : elle n'hésite plus à menacer de l'usage de la force. Manquant de soutien diplomatiques et surtout de la volonté de les mobiliser, la Chine n'a plus désormais qu'une autre option: en échange de quelques satisfactions de face et autres politesses politiques, alterner les discours moraux et les abstentions soigneusement calculées en réalité, donc, faire le dos rond en attendant des jours meilleurs pour elle et moins bons pour les Etats-Unis. Le calcul peut se révéler à terme payant. Dans ce dernier cas comme dans le premier, cependant, le coût intérieur de cette politique étrangère est considérable car elle passe 
pour une politique de lâcheté aux yeux d'une opinion passionnée et devient un objet de dissension au sein de l'élite.

Il faut donc conclure sur l'énigme. Les relations sino-américaines sont devenues l'un des points d'interrogation majeurs des relations internationales contemporaines. Elles portent en elles le meilleur et le pire, parce que, finalement, ces deux pays se sont rapprochés sans vraiment se comprendre.
$\mathbf{N} \cdot \mathbf{O} \cdot \mathrm{T} \cdot \mathrm{E} \cdot \mathrm{S}$

1. Michael Oksenberg, "American China Policy, 1971-1999, Managing a Major, Multifaceted Relationship", Paper, December 1999 ; Michael Oksenberg et Elizabeth Economy , China Joins the World, New York, 1999.

2. Henri Eyraud, "Chine et États-Unis face à face", Défense nationale, octobre 1996, pp. 19-29.

3. Un porte-avions américain prend en chasse un sous-marin chinois qui s'était approché de lui. Cf. LM 18 décembre 1994.

4. François Godement (ed), Chine-Etats-Unis, entre méfiance et pragmatisme, Paris, 2001, p. 13.

5. IHT (International Herald Tribune) 27-28 janvier 2001.

6. Parmi lesquelles quatre destroyers, des avions et, avec la collaboration d'autres pays occidentaux, huit sous-marins -tous matériels destinés à compenser les derniers achats chinois auprès de la Russie et à contrer un éventuel blocus maritime de Taiwan

7. BR (Beijing Review) 19 juillet 2001, p. 7 ; IHT 11-12 août 2001.

8. IHT 3 septembre 2001.

9. Voir notre article dans Critique internationale du premier trimestre 2002.

10. FEER (Far Eastern Economic Review) premier novembre 2001.

11. Voir l'interview du Ministre des Affaires 
étrangères chinois Tang Jiaxuan dans le Quotidien du peuple du 17 décembre 2001.

12. TE (The Economist), 23 février 2002.

13. AWSJ (Asian Wall Street Journal) 19-21 avril, IHT 2 mai 2002.

14. BR, 8 mars 2001, p. 7 ; LM (Le Monde) 20 avril 2001.

15. BR 12 juillet 2001, p. 9.

16. IHT 9 juillet 2001 ; BR 12 juillet 2001 p. 9. 17. Michael Oksenberg et Elizabeth Economy (1999); NW (Newsweek), 22 avril 1997, pp. 5056 ; LM 29 octobre 1997 ; FEER, 10 mai 2001, p. 22.

18. Chong-Ping Lin, "China' Students Abroad : Rates of Return", The American Enterprise, NovDec 1994, pp. 11-14; The Chronicle of Higher Education, 11 décembre 1998.

19. IHT, 28 mai 199

20. L (Libération), 6 juillet 1995.

21. FEER, 18 mai 1995, p. 23 ; communication de Jean-Louis Rallu au colloque de l'INED sur la démographie chinoise, Paris, janvier 2001.

22. Nikkei Weekly, 27 novembre 2000.

23. Stanley Rosen, "Chinese Youth in the Mi90's", Congrès de l'Association of Asian Studies, Hawaï, avril 1996. Ce sondage est en partie confirmé par un autre de la même époque, publié par BI, 21 octobre 1996.

24. IHT, 2 juillet 1999.

25. CI (Courrier InternationaI), 25 novembre 1993, p. 14.

26. Ibidem,, pp. 24 séq.

27. NW, 27 octobre 1997, p. 27; TE, 25 octobre
1997, p. 29.

28. IHT, 2 avril et 20 octobre 2000 ; FEER, 27 mai 1999, pp. 26-29 ; LM 26 mai 1999 ; FEER in CI, 23 décembre 1999-5 janvier 2000.

29. FEER 3 mai 2001, p. 18.

30. IHT 31 mai 2002. 